\title{
Can disk fragmentation leave signatures in spectral energy distributions?
}

\author{
O. V. Zakhozhay ${ }^{1, a}$ and E. I. Vorobyov ${ }^{2,3, b}$ \\ ${ }^{1}$ Main Astronomical Observatory, National Academy of Sciences of Ukraine, Kyiv, 03680, Ukraine \\ ${ }^{2}$ Institute of Astrophysics, The University of Vienna, Vienna, 1180, Austria \\ ${ }^{3}$ Research Institute of Physics, Southern Federal University, Rostov-on-Don, 344092, Russia
}

\begin{abstract}
In this article we show that massive and hot fragments forming in protostellar disks can leave characteristic signatures in spectral energy distributions (SEDs) of young stellar objects. This occurs when the fragment evaporates dust in its atmosphere, which results in a sharp drop in opacity and subsequent rise in the surface temperature of the fragment. These hot fragments can produce local maxima in the SEDs at 5-10 $\mu \mathrm{m}$.
\end{abstract}

\section{Introduction}

Observations of young protostellar disks and numerical modeling of disk formation and evolution both suggest that potostellar disks may be sufficiently massive to undergo gravitational fragmentation in the course of their evolution. Measurements of disk masses around young protostars embedded in parental cloud cores yield values that exceed $0.1 M_{\odot}$ (e.g. [4]). Numerical hydrodynamics simulations of disk formation indicate that such massive disks are gravitationally unstable and may fragment if disk cooling is sufficiently fast (e.g. [6]). The newly-formed fragments are in the sub-stellar mass regime and are considered as embryos of giant planets and brown dwarfs which may or may not evolve into finished objects ([7]). Directly observing these embryos is however challenging because it usually requires aperture masking interferometry to distinguish between the embryo and the rest of the disk. We therefore explore possibilities of using other indirect methods for detecting fragments forming within protostellar disks.

\section{Model description}

We base our model on numerical hydrodynamics simulations of [6], who studied the formation and evolution of protostellar disks subject to gravitational instability and fragmentation. The basic equations of hydrodynamics are solved on a polar grid in the thin-disk limit. This allows us to follow the gravitational collapse of a pre-stellar condensation (core) into the star plus disk formation stage and further to the T Tauri stage when most of the parental core has accreted onto the burgeoning disk. The following physical processes are taken into account: disk self-gravity via solution of the Poisson

\footnotetext{
ae-mail: zkholga@mail.ru

be-mail: eduard.vorobiev@univie.ac.at
}

This is an Open Access article distributed under the terms of the Creative Commons Attribution License 2.0, which permits unrestricted use, distribution, and reproduction in any medium, provided the original work is properly cited. 
integral and disk viscosity via $\alpha$-parameterization, radiative cooling from the disk surface, stellar and background irradiation, and also viscous and shock heating. More details can be found in ([6]).

\section{Spectral energy distribution}

To construct model SEDs, we split our model young stellar object (YSO) into three constituent parts: an accreting protostar, inner disk, and outer dynamic disk. The latter include a circumstellar disk and infalling envelope (if present).

We assume that our model YSO is viewed through the outflow cavity, which allows us to use disk surface densities and temperatures directly derived from numerical hydrodynamics modeling. Below, we explain in more detail the main ideas involved in calculating the model SEDs.

a) Outer dynamic disk. We use a solution to the one-dimensional radiation transfer equation to calculate the radiative flux density $F_{v}$ leaving each grid cell of the disk surface

$$
F_{v}^{\text {disk }}=\frac{S \cos \omega}{d^{2}} B_{v}\left(T_{\text {eff }}\right)\left(1-e^{-\Sigma \kappa_{v} \sec \gamma}\right),
$$
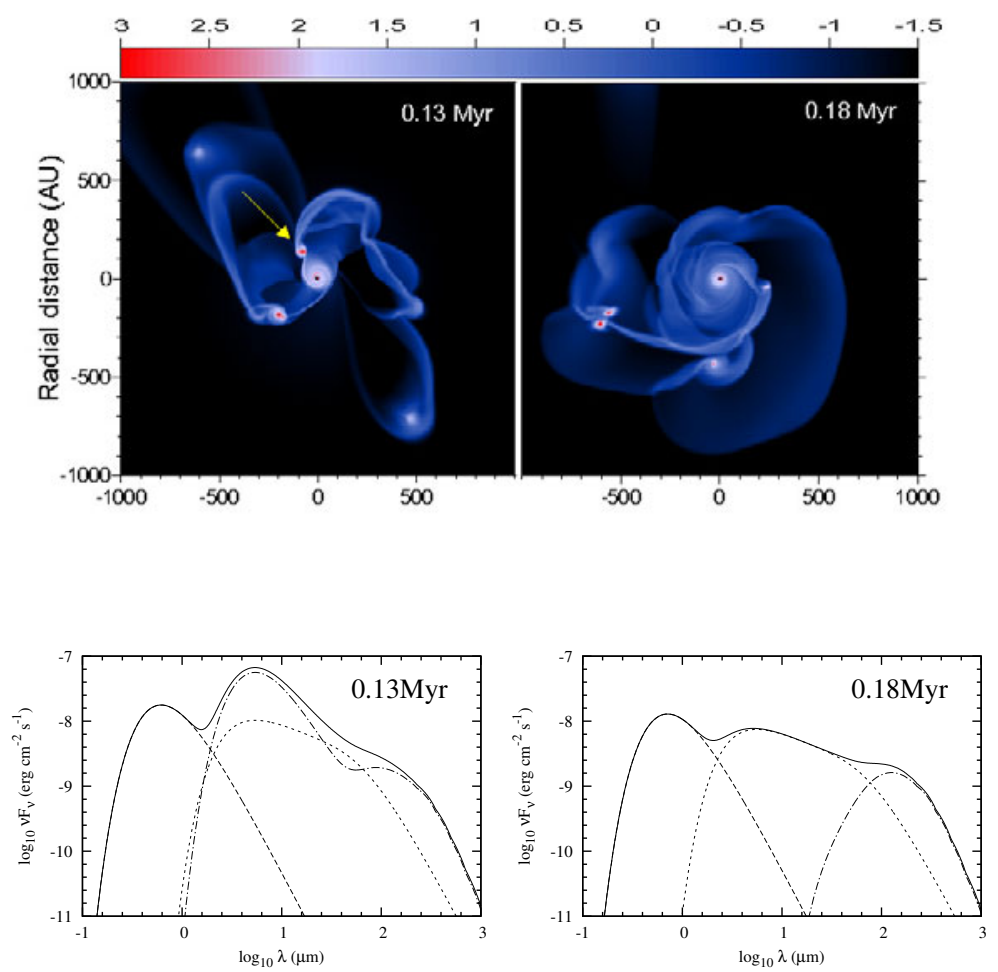

Figure 1. Top panels. Gas surface density maps of a fragmenting protostellar disk. The most massive fragment is shown by the arrow. Bottom panels. The corresponding SEDs. The total flux is shown by the solid line. 
where $B_{v}\left(T_{\text {eff }}\right)$ is the Plank function, $d=100 \mathrm{pc}$ is the distance to the considered object, $\omega$ is the normal angle between a specific grid cell of the disk surface and the line of sight, $\gamma$ is the inclination angle of the disk with respect to the observer, set to zero in this article and $S$ is the physical area of a specific grid cell. The frequency-dependent opacities $\kappa_{v}$ are taken from [5]. The effective temperature is the maximum of irradiation and surface temperatures, which are calculated as described in [6]. The total flux is found by summing inputs from all $(512 \times 512)$ individual grid cells of the disk.

b) Central accreting star. The radiative flux density from the protostar $F_{v}^{*}$ is calculated assuming a black-body radiation spectrum with effective temperature $T_{\mathrm{eff}}^{*}$

$$
F_{v}^{*}=\frac{\pi R_{*}^{2}}{d^{2}} B_{v}\left(T_{\mathrm{eff}}^{*}\right)
$$

where $R_{*}$ is the radius of the protostar and $T_{\text {eff }}^{*}$ is defined by both the accretion and photospheric luminosities as

$$
T_{\mathrm{eff}}^{*}=\left(\frac{L_{*, \mathrm{accr}}+L_{*, \mathrm{ph}}}{4 \pi R_{*}^{2} \sigma}\right)^{1 / 4},
$$

where $\sigma$ is the Stefan-Boltzmann constant, $L_{* \text {,accr }}$ and $L_{* \text {,ph }}$ are accretion and photospheric luminosities respectively. The accretion luminosity is calculated from the model's known accretion rate $\dot{M}$ onto the protostar, while the photospheric luminosity is taken from the pre-main sequence tracks of [2] for low-mass stars and brown dwarfs.

c) The inner disk. The effective temperature of the inner disk is calculated by extrapolating the corresponding azimuthally-averaged profiles of the outer dynamic disk down to the dust sublimation radius $R_{\text {d.s. }}$. The latter is calculated assuming the dust evaporation temperature on the inner age of the disk $T_{\text {d.s. }}=1500 \mathrm{~K}[3]$

$$
R_{\mathrm{d} . \mathrm{s}}=\sqrt{\frac{L_{*, \mathrm{accr}}+L_{*, \mathrm{ph}}}{16 \pi \sigma T_{\mathrm{d} . \mathrm{s} .}^{4}}} .
$$

The gas surface density distribution in the inner disk is calculated using the following relation

$$
\Sigma_{\text {in }}(r)=\Sigma_{0}\left(\frac{r}{r_{0}}\right)^{-p} .
$$

We chose the exponent $p=1.0$, since the inner disk is likely to be gravitationally stable [1]. The inner and outer disks are joined together at $r_{0}=10-20 \mathrm{AU}$, the latter value is chosen so as to make a smooth transition between the two disks. The typical gas surface density $\Sigma_{0}$ at $r_{0}$ is found using the least-squares fitting of the azimuthally averaged gas surface density in the outer dynamic disk.

The resulting profiles are then substituted into equation (1) to obtain the input from the inner disk to the total SED.

\section{Results}

The top panels in Figure 1 show the gas surface density maps of a protostellar disk formed as a result of gravitational collapse of a cloud core with initial mass $M_{\mathrm{c}}=1.2 M_{\odot}$. Only the inner $2000 \times 2000 \mathrm{AU}$ box is shown, the total computational area is ten times greater. The time (in Myr) is counted since the formation of the protostar. Disk fragmentation is evident in both panels. The masses and effective temperatures of the fragments are however different. The most massive and hot fragment shown with the arrow in the top-left panel of Fig. 1 has mass and effective temperature $0.06 M_{\odot}$ and $730 \mathrm{~K}$, respectively. The other fragment in the same panel is considerably lighter and cooler, with mass and 
temperature equal to $0.03 M_{\odot}$ and $74 \mathrm{~K}$, respectively. After $0.05 \mathrm{Myr}$ of evolution (top-right panel), the most massive fragment has migrated onto the star due to angular momentum exchange with spiral arms ([6]) and the remaining fragments have masses and temperatures not exceeding $0.03 M_{\odot}$ and $70 \mathrm{~K}$ respectively.

This change in the fragment properties is reflected in the corresponding SEDs presented in the bottom panels of Figure 1. Dashed, dotted, and dash-dotted lines show individual contributions from the protostar, inner disk, and outer dynamic disk, respectively, while the solid line is the total flux. The SED at $t=0.13$ Myr shows a well-defined peak at $\approx 5 \mu \mathrm{m}$, whereas the SED at $t=0.18$ Myr lacks such a feature. The occurrence of a peak at 5-10 $\mu \mathrm{m}$ may thus reflect the presence of a massive and hot fragment in the disk. The surface temperature of the fragment should exceed several hundred Kelvin. This can happen if the gas temperature in the fragment interiors exceeds the dust sublimation temperature and the opacity of the fragment drops considerably. Such fragments have not yet dissociated molecular hydrogen and therefore have not yet collapsed to a sub-stellar/planetary size, but have already evaporated dust in the atmosphere, thus revealing themselves as hot proto-brown dwarf embryos.

Acknowledgements. E.I.V. acknowledges support from the RFBR grant 11-02-92601-KO. O.V.Z. acknowledges travel support from the organizing committee of the ISPP workshop.

\section{References}

[1] S.V.W. Beckwith, A.I. Sargent, R.S. Chini, R. Güsten, AJ, 99, 924 (1990)

[2] F. D’Antona, I. Mazitelli, Memorie della Societa Astronomia Italiana, 68, 807 (1997)

[3] M.M. Dunham, N.J. Evans, S. Terebey, C.P. Dullemond, \& C.H. Young, ApJ 710, 470 (2010)

[4] J.K. Jorgensen, E.F. van Dishoeck, R. Visser, T.L. Bourke, D.J. Wilner, D. Lommen, M.R. Hogerheijde, P.C. Myers, A\&A 507, 861 (2009)

[5] V. Ossenkopf, Th. Henning, A\&A 291, 943 (1994)

[6] E.I. Vorobyov, S. Basu, ApJ 719, 1896 (2010)

[7] E.I. Vorobyov , S. Basu, ApJL 714, 133 (2010) 